\title{
PERFIL EPIDEMIOLÓGICO DOS CASOS DE LEISHMANIOSE VISCERAL EM SOBRAL-CE DE 2011 A 2015
}

\author{
EPIDEMIOLOGICAL PROFILE OF CASES OF VISCERAL LEISHMANIASIS IN
} SOBRAL, CEARÁ, BRAZIL, FROM 2011 TO 2015

PERFIL EPIDEMIOLÓGICO DE LOS CASOS DE LEISHMANIASIS VISCERAL EN SOBRAL, CEARÁ, BRASIL, DE 2011 A 2015

\author{
Natanael Aguiar de Sousa ${ }^{1}$ \\ Carlito Braga Linhares \\ Francisco Gustavo Barbosa Pires \\ Taynã Cesário Teixeira \\ Joab da Silva Lima \\ Maria do Livramento Oliveira Nascimento
}

Palavras-chave:

Leishmaniose Visceral; Epidemiologia; Saúde Pública.

Keywords:

Visceral Leishmaniasis; Epidemiology; Public Health.

Palabras clave:

Leishmaniasis Visceral; Epidemiología; Salud Pública.

Submetido: $10 / 09 / 2017$

Aprovado: 07/04/2018

Autor(a) para Correspondência: Natanael Aguiar de Sousa End: Rua Corina Dantas, 52, Bairro Derby - CEP: 62042-220, Sobral - CE E-mail:natan6521@gmail.com

\section{RESUMO}

A leishmaniose visceral (LV) é uma doença crônica causada por protozoário do gênero Leishmania. No Brasil, principalmente na região Nordeste, constitui um importante problema de saúde pública. Sobral-CE, em 2008, foi considerada área endêmica para transmissão dessa doença, por isso, o objetivo deste estudo foi descrever os aspectos e facetas epidemiológicas dos casos notificados de LV nesse município, no período de 2011 a 2015. A pesquisa foi desenvolvida com bases nos dados disponibilizados pelo Sistema de Informação de Agravos de Notificação (Sinan). As variáveis obtidas foram sexo, nivel de escolaridade e faixa etária, entre outras, que foram devidamente analisadas, organizadas, tabuladas e apresentadas em gráficos. Foi registrado um total de 161 casos no período. Adulto, homem, e com baixa escolaridade é o perfil dos mais afetados. Houve decréscimo no número absoluto de casos notificados, o que mostra que as medidas utilizadas em Sobral têm sido efetivas. Entretanto, mesmo com a redução do número de ocorrências, o município ainda é responsável por alta incidência no estado.

\footnotetext{
1. Aluno de Graduação em Medicina na Universidade Federal do Ceará (UFC). Sobral (CE), Brasil. E-mail: natan6521@gmail.com

2. Aluno de Graduação em Medicina na Universidade Federal do Ceará (UFC). Sobral (CE), Brasil. E-mail: carlitobraga22@gmail.com

3. Aluno de Graduação em Medicina na Universidade Federal do Ceará (UFC). Sobral (CE), Brasil. E-mail: gustavopires1322@gmail.com

4. Aluna de Graduação em Medicina na Universidade Federal do Ceará (UFC). Sobral (CE), Brasil. E-mail: tayteixeira14@gmail.com

5. Aluno de Graduação em Medicina na Universidade Federal do Ceará (UFC). Sobral (CE), Brasil. E-mail: jb.araticum@gmail.com

6. Bióloga graduada pela Universidade Estadual do Vale do Acaraú (UVA). Sobral (CE), Brasil. E-mail: mariaoliveeira2010@gmail.com
} 


\section{ABSTRACT}

Visceral leishmaniasis (VL) is a chronic disease caused by a protozoan of the genus Leishmania. In Brazil, mainly in the Northeastern region, this is a major public health issue. Sobral, Ceará, Brazil, in 2008, was regarded as an endemic area for transmission of the disease, so this study aimed to describe the aspects and epidemiological facets of reported cases of VL in this municipality, within the period from 2011 to 2015. The research was conducted having data provided by the Brazilian Information System for Notifiable Diseases (Sinan) as a basis. The variables obtained were sex, educational level, and age group, among others, which were duly analyzed, organized, tabulated, and presented in graphs. A total of 161 cases were registered within the period. Adult, man, and low educational level is the profile of the most affected individuals. There was a decrease in the absolute number of notified cases, something which shows that the measures used in Sobral have been effective. However, even with a decreased number of occurrences, the municipality is still responsible for a high incidence in the state.

\section{RESUMEN}

La leishmaniasis visceral (LV) es una enfermedad crónica causada por un protozoario del género Leishmania. En Brasil, principalmente en la región Nordeste, este es un importante problema de salud pública. Sobral, Ceará, Brasil, en 2008, fue considerada como un área endémica para transmisión de esta enfermedad, por lo que este estudio tuvo como objetivo describir los aspectos y las facetas epidemiológicas de los casos notificados de LV en este municipio, en el período de 2011 a 2015. La investigación se realizó teniendo como base los datos provistos por el Sistema Brasileño de Información de Enfermedades de Notificación (Sinan). Las variables obtenidas fueron sexo, nivel educativo y franja etaria, entre otras, que fueron debidamente analizadas, organizadas, tabuladas y presentadas en gráficos. Un total de 161 casos fueron registrados dentro del período. Adulto, hombre y bajo nivel educativo es el perfil de las personas más afectadas. Hubo una disminución en el número absoluto de casos notificados, algo que muestra que las medidas utilizadas en Sobral han sido efectivas. Sin embargo, incluso con un número reducido de ocurrencias, el municipio sigue siendo responsable por una alta incidencia en el estado.

\section{INTRODUÇÃO}

A leishmaniose visceral (LV), conhecida popularmente como calazar, é uma doença sistêmica, crônica, grave que pode acometer pele, mucosas e vísceras, dependendo da espécie do parasita e da resposta imune do hospedeiro. Causada por protozoário do gênero Leishmania, pertencente ao complexo Leishmania donovani, pode afetar o homem e outras espécies. No Brasil, o agente etiológico encontrado é a L. chagasi e o vetor da LV é o mosquito flebotomíneo fêmea da espécie Lutzomyia longipalpis, conhecido como mosquito palha. 0 parasita possui ciclo heteroxêmico, vivendo na forma promastigota no hospedeiro invertebrado (mosquito) e na forma amastigota no sistema monócito fagocitário do hospedeiro vertebrado (mamíferos). A infecção se inicia quando o flebotomíneo, regurgita no local da picada formas promastigotas presentes em sua faringe e seu esôfago, que são fagocitadas por células do sistema monocítico fagocitário do homem e transformadas em amastigotas (aflageladas) que se multiplicam no interior do vacúolo fagocitário do macrófago ${ }^{1}$.

Trata-se de uma doença espectral, cuja apresentação clínica varia de formas assintomáticas até o quadro clássico da parasitose, evidenciado pela presença de febre, anemia, hepatoesplenomegalia, além de tosse seca, leucopenia, pancitopenia, hipoalbuminemia e hipergamaglobulinemia. Outras manifestações clínicas se desenvolvem com a progressão da doença, em especial diarreia, icterícia, emagrecimento, vômito e edema periférico, que dificultam o diagnóstico diferencial com outras patologias, retardando sua identificação ${ }^{2,3}$.

0 diagnóstico pode ser feito pela clínica ou por pesquisa das formas amastigotas no interior de macrófagos ou monócitos, mediante exames de esfregaço de sangue periférico, aspirado de medula óssea (padrão ouro), aspirado esplênico (mais agressivo para o paciente), cultura da Leishmania e exames sorológicos, como ELISA e $\mathrm{K} 39^{1}$.

0 primeiro relato de LV no Brasil é de 1934, quando foram encontradas amastigotas de Leishmania em cortes histológicos de fígados de pessoas que morreram com suspeita de febre amarela. Somente 20 anos depois é que se registrou o primeiro surto da doença, em Sobral-CE, município do norte do Ceará. Em meados dos anos 1980, constatou-se uma transformação drástica na distribuição geográfica da 


\section{...Sobral se apresenta como o segundo município com maior número de casos notificados...}

LV. A doença, antes restrita às áreas rurais do Nordeste, avançou para outras regiões indenes, alcançando inclusive a periferia de grandes centros urbanos ${ }^{4}$.

Segundo os dados do Ministério da Saúde, de 2011 a 2015 foram notificados 16.927 casos no Brasil, sendo o Nordeste a região com o maior número de casos (8.714 no total), seguido pelo Norte (2.839), Sudeste (2.545), Centro-0este (1.302) e Sul (14); as regiões ignoradas nas fichas de notificação totalizaram 1.513 casos. No Nordeste, o Ceará foi o segundo em número de casos (2.146 notificações), ficando atrás somente do Maranhão (2.519 notificações). 0 coeficiente de incidência no Brasil ficou entre 1,6 a 2,0 por 100 mil habitantes no período, já o Ceará ficou com 3,8/100 mil habitantes em 2012 (menor incidência) e 6,3/100 mil habitantes em 2011 (maior incidência). A letalidade no Brasil ficou entre 6,6 e 7,8\%; já no Ceará foi de 5,8\% em 2013 e $8,7 \%$ em 2014, a menor e a maior taxa de letalidade no período, respectivamente. 0 número de óbitos nesse mesmo intervalo de tempo foi de 1.220 no Brasil e de 178 no Ceará ${ }^{\text {. }}$

No período de 2001 a 2010 foram registrados 173 casos desse agravo patológico em Sobral. Durante esse período, observou-se um crescimento do número de casos, com os primeiros notificados a partir do ano de 2002, 5 casos, alcançando um total de 40 em $2010^{6}$. 0 município foi considerado pelo Ministério da Saúde uma área endêmica para transmissão da LV7.

De acordo com o Informe Epidemiológico do Governo do Estado do Ceará, de 2014, Sobral se apresenta como o segundo município com maior número de casos notificados, ficando atrás apenas de Fortaleza (capital cearense), e classificado como município com transmissão intensa, segundo a estratificação de risco $^{8}$.

A LV afeta diferentes classes sociais e graus de escolaridade. Há relatos de casos em todas as faixas etárias, com destaque para o aumento proporcional de casos na população de 20 a 39 anos e, historicamente, ocorre maior proporção de casos no sexo masculino ${ }^{9}$.

Diversos fatores contribuíram para a expansão de áreas endêmicas com aparecimento de novos focos em consequência das transformações no ambiente, provocadas pelo intenso processo migratório, por agressões ao ambiente, por pressões econômicas ou sociais, a pauperização decorrente de distorções na distribuição de renda, o crescente processo de urbanização, o esvaziamento rural e as secas periódicas ${ }^{6}$.

A estratégia de controle está centrada na identificação e eliminação dos reservatórios, principalmente o cão, aplicação de inseticidas para eliminação do vetor, e diagnóstico e tratamento adequados dos casos notificados. Maior conhecimento científico sobre o papel específico de cada elemento da cadeia de transmissão (agente etiológico, inseto transmissor, homem e reservatórios silvestres e domésticos) representa um dos maiores desafios para o aprimoramento das estratégias de controle ${ }^{8}$.

0 conhecimento acerca das características epidemiológicas da LV em Sobral, município com elevado número de casos notificados e de óbitos, pode ajudar as autoridades governamentais e as unidades de saúde na elaboração de estratégias para prevenção, controle e tratamento do agravo. Logo, o objetivo deste estudo foi descrever os aspectos epidemiológicos dos casos de LV notificados em Sobral, no período de 2011 a 2015.

\section{METODOLOGIA}

A pesquisa documental foi fundamentada nos dados sobre os casos de LV disponiveis no Sistema Nacional de Notificações e Agravos (Sinan) para o município de Sobral de 2011 a 2015.

Sobral, localiza-se a $235 \mathrm{~km}$ de Fortaleza e liga-se à capital do estado pela BR- 222 - que também liga o Ceará ao Piauí, ao Maranhão e ao Pará. Suas coordenadas geográficas são $3^{\circ} 41^{\prime} 10^{\prime \prime}$ de latitude e $40^{\circ} 10^{\prime} 59^{\prime \prime}$ de longitude. Segundo o Instituto Brasileiro de Geografia e Estatística (IBGE), em 2016, a área de unidade territorial era de $2.122,897 \mathrm{~km}^{2}$ e a população estimada para o ano de 2017 foi de 205.529 habitantes. Localizado no norte do Ceará, o município faz limite ao norte com Massapê, Santana do Acaraú e Meruoca; ao sul faz limite com Santa Quitéria, Groaíras e Cariré; a leste faz limite com Itapipoca, Irauçuba e Canindé; e a oeste faz limite com Coreaú, Mucambo e Alcântaras. Seu clima é quente e seco, com temperatura média de $30^{\circ} \mathrm{C}^{10,11}$. 
Foram analisadas as informações referentes aos indivíduos que contraíram LV, registrados na base de dados do Sinan, como já citado, disponível para consulta gratuita em seu site. As principais variáveis obtidas para as informações epidemiológicas foram: faixa etária, sexo, nível de escolaridade, tipo de entrada no sistema de saúde, evolução da doença em cada indivíduo para traçar o perfil epidemiológico da doença e incidência da doença em Sobral de 2011 a 2015.

0 Sinan é suprido, principalmente, pela notificação e investigação de casos de doenças e agravos que constam da lista nacional de doenças de notificação compulsória ${ }^{12}$, mas é facultado a estados e municípios incluir outros problemas de saúde de sua região e tem por objetivo o registro e processamento dos dados sobre agravos de notificação em todo o território nacional, fornecendo informações para análise do perfil da morbidade e contribuindo, dessa forma, para a tomada de decisões em nível municipal, estadual e federal ${ }^{13}$.

Segundo o Sinan, os dados obtidos para o ano de 2011 foram atualizados em 29/08/2012, os de 2012 em 12/08/2013, os de 2013 em 25/08/2014, os de 2014 em 19/08/2015 e os de 2015 ainda são parciais (sujeitos a revisão) ${ }^{14}$.

Os resultados foram tabulados e convertidos em gráficos para sintetizar e reunir informações de modo organizado e conciso, facilitando a investigação. Essa ferramenta proporciona uma análise mais complexa, incluindo a estatística descritiva, o teste da significância e a análise da variância. 0s dados foram organizados e processados no programa computacional Microsoft Excel, versão 2007.

\section{RESULTADOS E DISCUSSÃO}

Segundo os dados coletados no Sinan, um total de 161 casos de LV foram notificados no período de 2011 a 2015 em Sobral. Ao contrário da epidemiologia registrada na década anterior, quando os dados apontavam um tendência crescente, com aumento no número de casos a cada ano, no período em estudo, os dados indicam uma tendência decrescente, com diminuição no número de casos notificados, tendo ápice em 2011, com 53 casos (32,9\% do total); 2012 apresentou 38 casos $(23,6 \%)$, ao passo que 2013 teve $28(17,3 \%), 2014$ teve $25(15,5 \%)$ e 2015 teve 17 casos $(10,5 \%)^{6}$.

Essa diminuição pode demonstrar que as medidas de prevenção da doença, principalmente contra o vetor, têm mostrado resultados satisfatórios. Acredita-se, ainda, que esses números possam ser mais elevados ao considerar a subnotificação de casos.

Em comparação com o estado do Ceará, Sobral mostrou taxas de incidência bem mais elevadas, com $27,8 / 100$ mil habitantes em 2011, subindo para $8,4 / 100$ mil habitantes em 2015. Já o estado permaneceu com seus números seguindo um padrão linear, sem decréscimo acentuado, com 7,2/100 mil habitantes em 2011 e 6,4/100 mil habitantes em 2015 (Figura 1). Percebe-se que o município contribuiu de modo significativo para o aumento da incidência de LV no estado dentro do período em estudo.

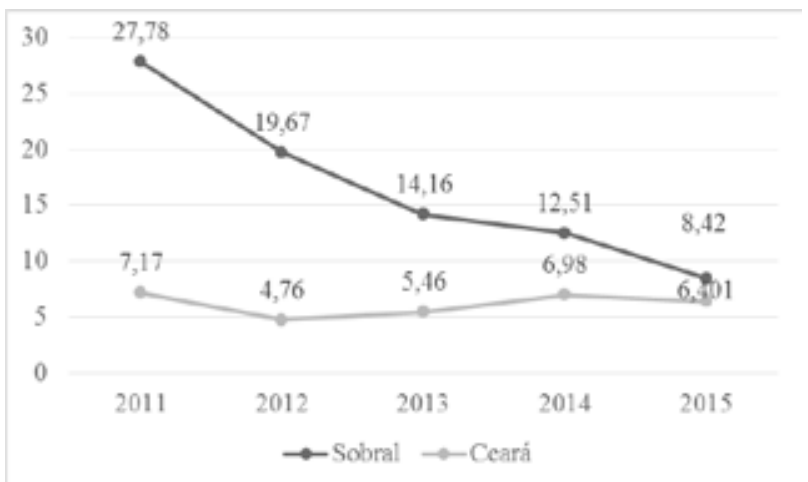

Figura 1. Coeficientes de incidência (por $100 \mathrm{mil}$ habitantes) de leishmaniose visceral no município de Sobral e no estado do Ceará, de 2011 a 2015. Fonte: Elaborada pelos autores.

Analisando a variável faixa etária (Figura 2), entre os adultos predomina a faixa dos 20 aos 39 anos (41 casos) e entre as crianças predomina a faixa de 1 a 4 anos (38 casos). Isso indica que são esses os grupos mais vulneráveis à afecção.

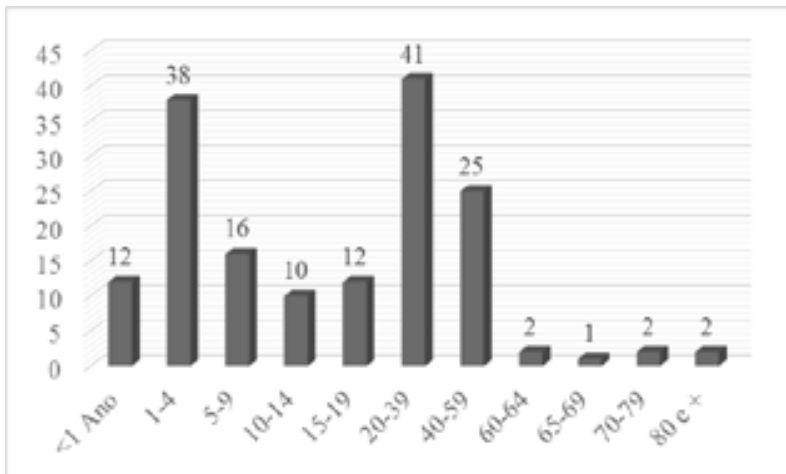

Figura 2. Número de casos notificados de leishmaniose visceral, segundo faixa etária, em Sobral, de 2011 a 2015.

Fonte: Elaborada pelos autores. 
A ocorrência da doença em adultos pode ser justificada por sua maior exposição aos flebotomíneos vetores, por serem considerados pertencentes ao grupo dos indivíduos economicamente ativos. Já a frequência em crianças apontada neste estudo também foi observada em um estudo realizado no Rio de Janeiro, que associou tal achado ao contato mais frequente das crianças com animais, além de sua maior carência nutricional e de seu estado imunológico ainda em formação ${ }^{15}$. Um resultado semelhante foi encontrado em um estudo realizado no Ceará, referente ao período de 2007 a 2011, e em outro estudo semelhante realizado em Sobral, referente ao período de 2001 a $2010^{6,16}$.

No que se refere ao sexo (Figura 3), em Sobral, a LV acomete com maior frequência a população masculina em todos os anos do período em estudo: $68,9 \%$ dos acometidos no período são homens. A doença pode atingir todas as idades e ambos os sexos, porém, a prevalência da doença nos homens pode ser justificada pela maior exposição aos vetores flebotomíneos e não por maior suscetibilidade ${ }^{17}$.

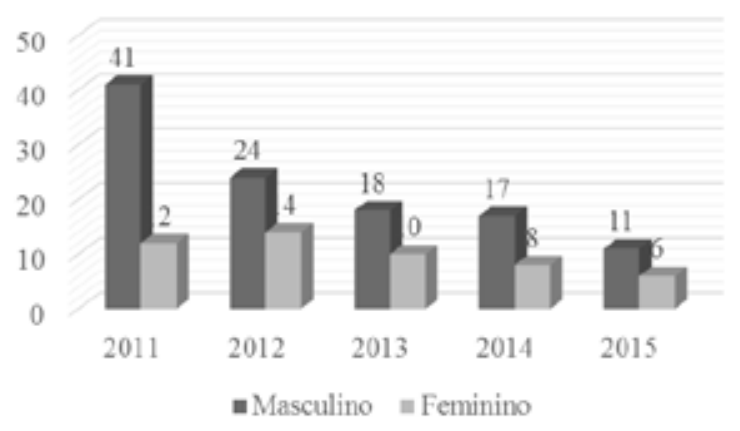

Figura 3. Número de casos notificados de leishmaniose visceral, segundo sexo, em Sobral, de 2011 a 2015. Fonte: Elaborada pelos autores.

Em relação ao grau de instrução (Figura 4), percebe-se que o número de indivíduos com alta escolaridade (Ensino Médio completo e Ensino Superior completo) e acometidos pela doença é baixo $(4,9 \%$ do total) - provavelmente, isso se deve ao maior acesso às informações sobre medidas preventivas. Já os indivíduos com baixa escolaridade e os analfabetos compõem a maioria dos doentes, evidenciando que a alta prevalência da LV nesse grupo pode estar associada a menor educação em saúde e menor potencial de controle epidemiológico. No entanto, os graus de instrução considerados ignorados ou em branco e os que não se aplicam, neste estudo, que juntos correspondem a $63,3 \%$ do total, podem ser justificados, em parte, pelo fato de que $31 \%$ dos casos de acometidos pela LV notificados correspondem a menores de 4 anos de idade, os quais ainda não frequentam o ambiente escolar. Resultados semelhantes a esses foram obtidos por estudos realizados em Sobral, no período de 2001 a 2010, e em Paracutu-MG, de 2007 a $2010^{6,17}$.

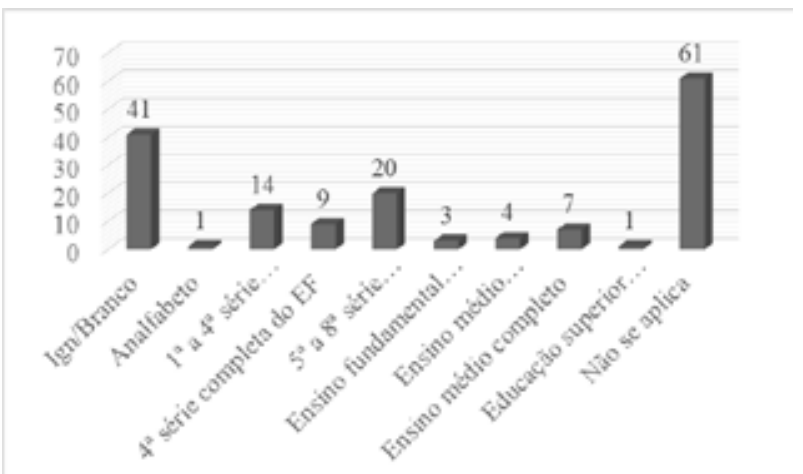

Figura 4. Número de casos notificados de leishmaniose visceral, segundo nível de escolaridade, em Sobral, de 2011 a 2015.

Fonte: Elaborada pelos autores.

Dentre os indivíduos com LV no período em estudo, $128(79,5 \%)$ obtiveram a cura como desfecho, 10 evoluíram para óbito e a letalidade observada nesse período variou de $3,5 \%$ a $8,0 \%$, com média de $6,2 \%$, ficando abaixo da média tanto do país $(6,8 \%)$ como do estado $(7,8 \%)$ no mesmo intervalo de tempo. Dentre os acometidos, $3(1,8 \%)$ evoluíram para óbito por outras causas, $3(1,8 \%)$ abandonaram o tratamento e o vínculo com o sistema de saúde, 16 (9,9\%) foram transferidos do município e $1(0,6 \%)$ foi considerado ignorado ou branco.

Essa grande proporção de cura nos casos notificados pode ser justificada pelo início precoce do tratamento e pelo bom preparo dos serviços de saúde do município, com profissionais preparados, testes laboratoriais e medicamentos para melhor atender a toda a demanda.

Por outro lado, $6(3,7 \%)$ dos casos de 2011 a 2015 foram notificados como recidivas. Isso pode ser explicado por ineficácia do tratamento, abandono e diminuição da capacidade de resposta do sistema imunológico da pessoa doente, além de comorbidades associadas à $L^{5}$. Entretanto, $117(72,6 \%)$ foram casos novos e $38(23,6 \%)$ foram casos notificados como transferidos de outros municípios (Figura 5 ). 


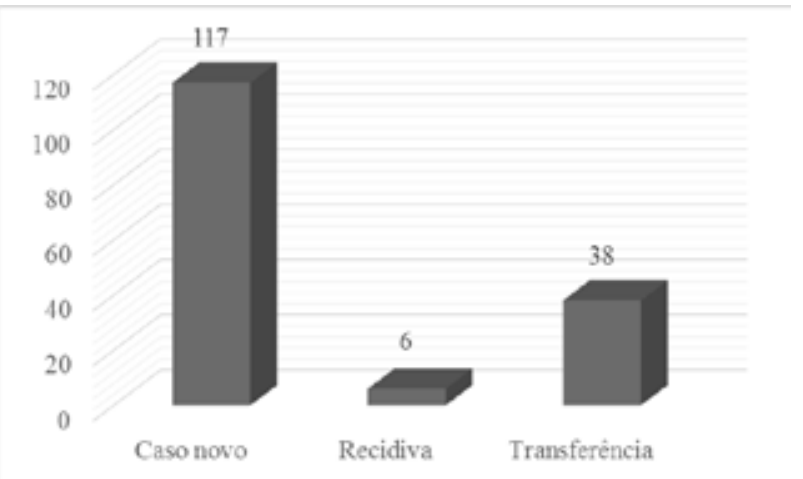

Figura 5. Número de casos notificados de leishmaniose visceral, segundo tipo de entrada, em Sobral, de 2011 a 2015.

Fonte: Elaborada pelos autores.

Considera-se que esses resultados apresentados, principalmente o decréscimo da taxa de incidência de LV e o aumento das taxas de cura, refletem a organização dos serviços na Atenção Primária à Saúde (APS) do município, principalmente com a implantação e expansão das ações das equipes da Estratégia Saúde da Família (ESF), que funcionam como porta de entrada do Sistema Único de Saúde (SUS). Entretanto, quando se compara ao estado e a outras cidades, os índices ainda se mostram elevados, sendo necessária a continuação e o aperfeiçoamento do acompanhamento dos acometidos e do trabalho de prevenção e de promoção da saúde já adotados.

\section{CONCLUSÃO}

Este estudo possibilitou a avaliação epidemiológica da incidência de LV em Sobral, no período de 2011 a 2015. Os dados que embasaram esta pesquisa avaliaram diferentes características da populaçãoalvo, comparando-as com períodos anteriores.

0 s resultados obtidos indicaram uma significativa redução do número de casos notificados anualmente, seguindo uma tendência de aproximação à média estadual e nacional. Observou-se, ainda, predominância do surgimento de casos em grupos específicos, como sexo masculino, classes socioeconômicas mais baixas e menores graus de escolaridade.

Assim, esta pesquisa possibilitou observar que, com o decréscimo no número de casos a cada ano, as medidas que têm sido adotadas no combate à doença no município se mostram efetivas. Ainda assim, sua média segue superior tanto ao nível estadual como nacional, o que indica a necessidade de manter e até intensificar tais medidas.

Percebe-se, também, que algumas características relacionadas à incidência no perfil da população ainda persistem, com alguns grupos se mostrando mais suscetíveis. Tal fato reforça a necessidade de uma abordagem mais direcionada e diferenciada a esses grupos, por parte do município, de modo a acentuar a redução dos índices de contração da doença.

\section{CONTRIBUIÇÃO DOS AUTORES}

Natanael Aguiar de Sousa contribuiu com 0 delineamento e a realização da pesquisa e a estruturação e redação do manuscrito. Carlito Braga Linhares e Francisco Gustavo Barbosa Pires contribuíram com a redação do manuscrito. Taynã Cesário Teixeira contribuiu com a realização da pesquisa. Joab da Silva Lima e Maria do Livramento Oliveira Nascimento contribuíram com a revisão crítica do manuscrito.

\section{REFERÊNCIAS}

1. Neves DP. Parasitologia humana. 13. ed. São Paulo: Atheneu; 2016.

2. Pastorino AC, Jacob CMA, Oselka GW, Carneiro-Sampaio MMS. Leishmaniose visceral: aspectos clínicos e laboratoriais. J Pediatr [serial on the internet]. 2002 [cited 2018 May 14];78(2):120-7. Available from: http://www. scielo.br/pdf/\%0D/jped/v78n2/v78n2a10.pdf

3. Pedrosa CMS, Rocha EMM. Aspectos clínicos e epidemiológicos da leishmaniose visceral em menores de 15 anos procedentes de Alagoas, Brasil. Rev Soc Bras Med Trop [serial on the internet]. 2004 [cited 2018 May 14];37(4):3004. Available from: http://www.scielo.br/pdf/rsbmt/ v37n4/21183.pdf

4. Gontijo CMF, Melo NM. Leishmaniose visceral no Brasil: quadro atual, desafios e perspectivas. Rev Bras Epidemiol [serial on the internet]. 2004 [cited 2017 Jul 17];7(3):33849. Available from: http://www.scielo.br/pdf/rbepid/ v7n3/11.pdf

5. Portal da Saúde [homepage on the internet]. Leishmaniose visceral: situação epidemiológica/dados. c2017 [cited 2017 Jul 17]. Available from: http:// portalsaude.saude.gov.br/index.php/o-ministerio/ principal/leia-mais-o-ministerio/726-secretaria-svs/ vigilancia-de-a-a-z/leishmaniose-visceral-lv/11334situacao-epidemiologica-dados

6. Silva OI, Vieira RDN, Timbó PEB. Perfil epidemiológico dos casos de leishmaniose visceral em Sobral, Ceará no período de 2001 a 2010. Sanare (Sobral, Online) [serial on 
the internet]. 2013 [cited 2017 Jul 17];12(1):13-9. Available from: file:///D:/323-624-1-SM\%20(2).pdf

7. Diário do Nordeste [homepage on the internet]. Cidade de Sobral é área endêmica para o calazar. 2008 [cited 2017 Jul 17]. Available from: http://diariodonordeste. verdesmares.com.br/cadernos/regional/cidade-de-sobral-earea-endemica-para-o-calazar-1.497175

8. Ceará (Estado). Informe epidemiológico: leishmaniose [document on the internet]. 2014 [cited 2017 Jul 17]. Available from: www.saude.ce.gov.br/index.php/boletins $\% 2$ 53Fdownload\%253D1559\%25253Ainforme-epidemiologicoleishmaniose-agosto-de-2014\%

9. Ceará (Estado). Boletim epidemiológico: leishmaniose visceral [document on the internet]. 2016 [cited $2017 \mathrm{Jul}$ 17]. Available from: http://www.saude.ce.gov.br/index. php/boletins?download $=2750 \% 3$ Aboletim-epidemiologicoleishmaniose-visceral-20-de-outubro-de-2016

10. Sobral (Município). A cidade: história [document on the internet]. c2017 [cited 2017 Jul 19]. Available from: http:// www.sobral.ce.gov.br/site novo/index.php/a-cidade/ historia

11. Instituto Brasileiro de Geografia e Estatística [homepage on the internet]. Cidades: Sobral c2017 [cited 2017 Sep 8]. Available from: http://cidades.ibge.gov.br/xtras/perfil. php? codmun $=231290$

12. Brasil. Portaria n. 204, de 17 de fevereiro de 2016. Define a Lista Nacional de Notificação Compulsória de doenças, agravos e eventos de saúde pública nos serviços de saúde públicos e privados em todo o território nacional, nos termos do anexo, e dá outras providências [document on the internet]. 2016 [cited 2018 May 14]. Available from: http:// bvsms.saude.gov.br/bvs/saudelegis/gm/2016/ prt0204 1702 2016.html

13. Instituto Brasileiro de Geografia e Estatística [homepage on the internet]. Comitê de Estatísticas Sociais: Sistema de Informação de Agravos de Notificação - Sinan. c2017 [cited $2017 \mathrm{Jul}$ 19]. Available from: http://ces.ibge.gov.br/basede-dados/metadados/ministerio-da-saude/sistema-deinformacoes-de-agravos-de-notificacao-sinan.html

14. Sistema de Informação de Agravos de Notificação [homepage on the internet]. Sinan. c2017 [cited 2017 Jul 19]. Available from: http://tabnet.datasus.gov.br/cgi/ deftohtm.exe?sinannet/cnv/leishvce.def

15. Marzochi MCA, Fagundes A, Andrade MV, Souza MB, Madeira MF, Mouta-Confort E, et al. Visceral leishmaniasis in Rio de Janeiro, Brazil: ecoepidemiological aspects and control. Rev Soc Bras Med Trop [serial on the internet] 2009 [cited 2017 Jul 18];42(5):52-63. Available from: http:// www.scielo.br/pdf/rsbmt/v42n5/17.pdf

16. Calvacante IJM, Vale MR. Aspectos epidemiológicos da leishmaniose visceral (calazar) no Ceará no período de 2007 a 2011. Rev Bras Epidemiol [serial on the internet]. 2014 [cited 2017 Jul 20];17(4):911-24. Available from: https:// pdfs.semanticscholar.org/d5f8/3434a00c3bc78c36149db476 b85a18f4ff5f.pdf

17. Oliveira EN, Pimenta AM. Perfil epidemiológico da leishmaniose visceral no município de Paracatu, MG no período de 2007 a 2010. REME Rev Min Enferm [serial on the internet]. 2014;18(2):365-75. Available from: file:///D:/ v18n2a09.pdf
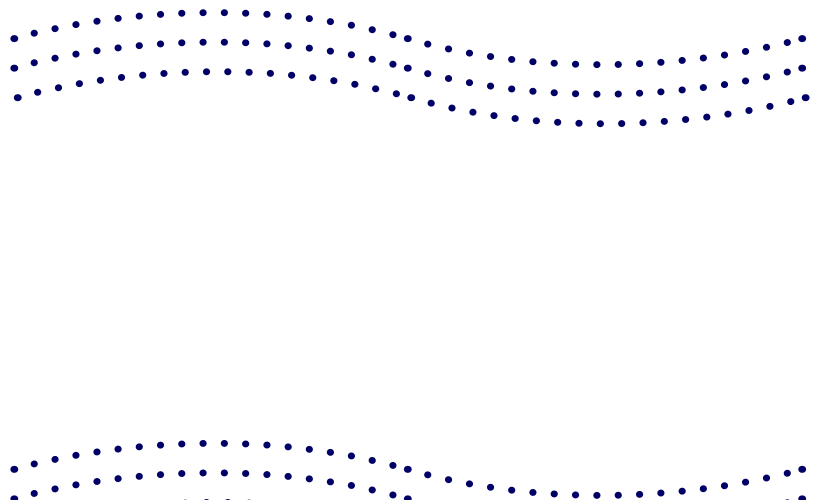
$\ldots \ldots \ldots \ldots \ldots \ldots \ldots \ldots$ $\ldots \ldots \ldots \ldots \ldots \ldots \ldots$ $\cdots \ldots \ldots \ldots \ldots$
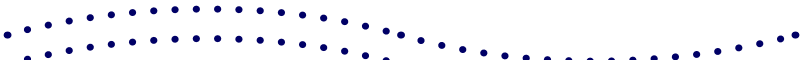
$\ldots \ldots \ldots \ldots \ldots \ldots \ldots$ $\ldots \ldots \ldots \ldots \ldots$
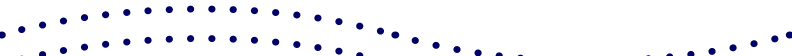
$\ldots \ldots \ldots \ldots \ldots \ldots \ldots \ldots$ $\ldots \ldots \ldots \ldots \ldots \ldots \ldots$

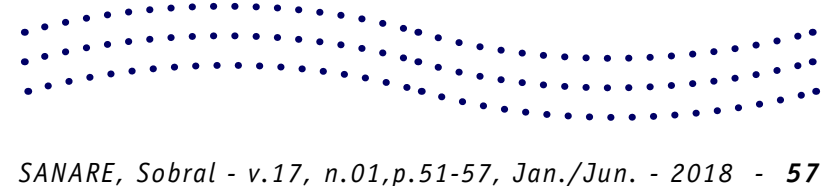

